\title{
Design of Doubly-Complementary IIR Digital Filters Using a Single Complex Allpass Filter, With Multirate Applications
}

\author{
P. P. VAIDYANATHAN, MEMBER, IEEe, PHILLIP A. REGALIA, STUDENT MEMBER, IEEe, \\ AND SANJIT K. MITRA, FELLOW, IEEE
}

\begin{abstract}
It is shown that a large class of real-coefficient doubly-complementary IIR transfer function pairs can be implemented by means of a single complex allpass filter. For a real input sequence, the real part of the output sequence corresponds to the output of one of the transfer functions $G(z)$ (for example, lowpass), whereas the imaginary part of the output sequence corresponds to its "complementary" filter $H(z)$ (for example, highpass). The resulting implementation is structurally lossless, and hence the implementations of $G(z)$ and $H(z)$ have very low passband sensitivity. Numerical design examples are included, and a typical numerical example shows that the new implementation with 4 bits per multiplier is considerably better than a direct form implementation with 9 bits per multiplier. Multirate filter bank applications (quadrature mirror filtering) are outlined.
\end{abstract}

\section{INTRODUCTION}

$\mathrm{T}$ HERE EXIST a number of digital filter structures with low passband sensitivity. These include wave-digital filters [1]-[5], orthogonal filters [6]-[8], and other structures based on second-order sections [9], [10]. Some of these structures have an inherent passivity [1]-[8] and, as a result, can be designed to be free from parasitic oscillations (limit cycles) [11], [12].

A unified viewpoint of several low-sensitivity structures can be obtained in terms of the concept of "structural boundedness" [13]: Thus, let $G(z)$ be a stable digital filter transfer function that is real-valued for real $z$ and satisfying $\left|G\left(e^{j \omega}\right)\right| \leqslant 1$ for all $\omega$. Such transfer functions are said to be "bounded real" (BR). When the BR function has $\left|G\left(e^{j \omega}\right)\right|=1$ for all $\omega$, it is called a "lossless bounded real" (LBR) transfer function. Thus, a stable allpass function with real coefficients is LBR. An implementation of a BR transfer function is said to be "structurally bounded" or "structurally passive" if the transfer function continues to be BR in spite of parameter quantizations. Such implementations are known to exhibit very low passband sensitivity [13]-[15], for the same reason that the structures in

Manuscript received May 26, 1986; revised October 17, 1986. This work was supported in part by a University of California MICRO Grant, with matching funds from Intel Corp., Rockwell Corp., and LSI Logic Corp., in part by the National Science Foundation under Grants ECS 82-18310, ECS 84-04245, and ECS 85-08017, and in part by a Caltech Programs in Advanced Technology Grant.

P. P. Vaidyanathan is with the Department of Electrical Engineering, California Institute of Technology, Pasadena, CA 91125.

P. Regalia and S. K. Mitra are with the Department of Electrical and Computer Engineering, University of California, Santa Barbara, CA 93106.

IEEE Log Numbcr 8612932.

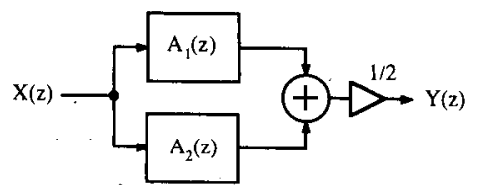

Fig. 1. Parallel connection of two allpass sections.

[1]-[8] have low sensitivity. In addition, structurally pas sive implementations can be tailored to be such that ther are no limit cycles [16], [17].

There exists a certain class of BR transfer function which can be implemented in the form of a paralle interconnection of two allpass filters (Fig. 1) with rea coefficients. This observation has been made in connectio with wave filters by Fettweis [4], and by other authors in the recent past [15], [18], [28]. Such realizations satisfy th structural-boundedness property and hence exhibit ver low passband sensitivity [15]. In addition, the allpass fund tions can be implemented as a cascade of lossless lattic structures [19], [20], thereby suppressing zero-input limi cycles [12]. The key point concerning the sum-of-allpas implementation is that each allpass function can be imple mented such that it continues to remain allpass (i.e., LBR in spite of coefficient quantization. Such allpass implemen tations are called structurally lossless. Accordingly, th overall transfer function $G(z)$ remains passive in spite o coefficient quantization. This leads to low coefficien sensitivity; details can be found in [15].

There are certain conditions which a BR transfer fund tion $G(z)$ has to satisfy, so that it can be implemented a in Fig. 1. In particular, let $G(z)$ be an $N$ th-order low-pas transfer function of the form

$$
G(z)=\frac{P(z)}{D(z)}
$$

where

$$
\begin{aligned}
& P(z)=p_{0}+p_{1} z^{-1}+\cdots+p_{N} z^{-N} \\
& D(z)=1+d_{1} z^{-1}+\cdots+d_{N} z^{-N} .
\end{aligned}
$$

Here $p_{n}, d_{n}$ are real and $P(z)$ is a symmetric (or mirror image) polynomial, i.e.,

$$
P\left(z^{-1}\right)=z^{N} P(z)
$$




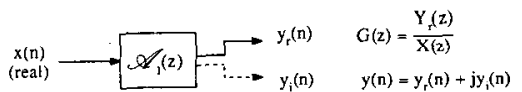

Fig. 2. Implementation of $G(z)$ by means of a single complex allpass function. Solid lines indicate real parts and broken lines indicate imaginary parts.

It can be shown that $N$ has to be odd so that an implementation in the form of Fig. 1 is feasible. For example, odd-ordered low-pass digital Butterworth, Chebyshev, and elliptic filters can be implemented as in Fig. 1. One purpose of this paper is to propose a modification to the structure of Fig. 1 for the case of an even-order low-pass function $G(z)$. In Section II, we outline the decomposition of $G(z)$ into a sum of allpass functions $\mathscr{A}_{1}(z)$ and $\mathscr{A}_{2}(z)$ with complex coefficients. The decomposition is such that the coefficients of $\mathscr{A}_{2}(z)$ are complex conjugates of the coefficients of $\mathscr{A}_{1}(z)$. Accordingly, for real inputs, it is only required to implement a complex filter $\mathscr{A}_{1}(z)$ and retain the real part $y_{r}(n)$ of the output sequence $y(n)$ in order to obtain an implementation of $G(z)$, as illustrated in Fig. 2. Moreover, it can be shown that the imaginary part $y_{i}(n)$ of the output sequence $y(n)$ corresponds to a "complementary" filter, i.e., if we define

$$
H(z)=\frac{Y_{i}(z)}{X(z)}
$$

then the following relation holds:

$$
\left|G\left(e^{j \omega}\right)\right|^{2}+\left|H\left(e^{j \omega}\right)\right|^{2}=1 .
$$

In other words, $G(z)$ and $H(z)$ constitute a power-complementary pair. In particular, if $G(z)$ has a low-pass response, then $H(z)$ is highpass.

Section II presents the details of such an allpass decomposition. Section III includes a design example and computer-aided simulation results that verify the low passband sensitivity property. Structures for implementing the complex allpass functions are included in Section IV. Finally, Section $\mathrm{V}$ outlines an application of the complementary pair $\{G(z), H(z)\}$ in multirate signal processing, viz., quadrature-mirror filtering (QMF banks); we show how the complementary pair of Section II is ideally suited for splitting a signal into two bands, decimating, and then recombining, with no aliasing error and no amplitude distortion error. These results are presented for both the schemes of Figs. 1 and 2. Efficient low-sensitivity polyphase structures based on allpass filters are also indicated in Section $\mathrm{V}$ for some of the QMF banks.

\section{Decomposition of a BR Function into a Sum of Two Complex Allpass Sections}

Let $G(z)=P(z) / D(z)$ be an $N$ th-order BR function as in (1) given in minimal form (i.e., no common factors between $P(z)$ and $D(z)$ ). Let $P(z)$ be a symmetric polynomial satisfying (2). Consider a BR function $H(z)$ with the same denominator $D(z)$

$$
H(z)=\frac{Q(z)}{D(z)}=\frac{q_{0}+q_{1} z^{-1}+\cdots+q_{N} z^{-N}}{1+d_{1} z^{-1}+\cdots+d_{N} z^{-N}}
$$
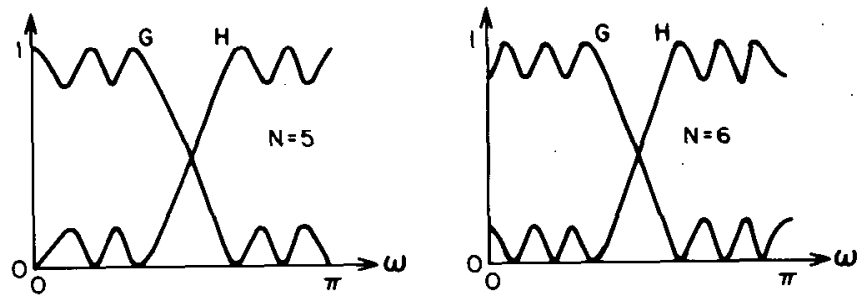

Fig. 3. Typical complementary pairs (the squares of the magnitude are being plotted).

such that $\{G(z), H(z)\}$ form a power-complementary pair, i.e., satisfy (4). Such $H(z)$ can always be constructed by finding a spectral factor $Q\left(e^{j \omega}\right)$ of the positive function

$$
\left|D\left(e^{j \omega}\right)\right|^{2}-\left|P\left(e^{j \omega}\right)\right|^{2} \text {. }
$$

In many filtering applications, it is possible to find a spectral factor $Q(z)$ such that $Q(z)$ is symmetric or antisymmetric. This happens, in particular, if $H(z)$ has all its transmission zeros on the unit circle. Fig. 3 depicts typical response curves for $N=5$ and 6 . Notice that for even $N$, there is no transmission zero for $H(z)$ at $z=1$, whereas for odd $N$, there is such a transmission zero. Accordingly, $Q(z)$ is symmetric for the case of even $N$, and antisymmetric when $N$ is odd.

When $P(z)$ is symmetric and $Q(z)$ antisymmetric, it can be shown [15] that $G(z)$ and $H(z)$ can be written in terms of stable allpass functions $A_{1}(z)$ and $A_{2}(z)$ with real coefficients as follows:

$$
\begin{aligned}
& G(z)=\frac{1}{2}\left[A_{1}(z)+A_{2}(z)\right] \\
& H(z)=\frac{1}{2}\left[A_{1}(z)-A_{2}(z)\right]
\end{aligned}
$$

where $A_{1}(z)$ and $A_{2}(z)$ are given by

$$
\begin{aligned}
& A_{1}(z)=\prod_{k=r+1}^{N}\left(\frac{z^{-1}-z_{k}^{-1}}{1-z^{-1} z_{k}^{*-1}}\right) \\
& A_{2}(z)=\prod_{k=1}^{r}\left(\frac{z^{-1}-z_{k}}{1-z^{-1} z_{k}^{*}}\right) .
\end{aligned}
$$

The quantities $z_{k}$ in (9a) are the zeros (possibly complex) of $P(z)+Q(z)$. Of these, $\left\{z_{1}, z_{2}, \cdots, z_{r}\right\}$ lie inside the unit circle of the $z$-plane, whereas $z_{r+1}, z_{r+2}, \cdots, z_{N}$ lie outside the unit circle. These zeros (and hence $r$ ) are found as described in [15]. It can also be shown [15] that $\left\{z_{1}, z_{2}, \cdots, z_{r}, 1 / z_{r+1}, \cdots, 1 / z_{N}\right\}$ are precisely the zeros of $D(z)$ (which is the denominator common to both $G(z)$ and $H(z)$ ). Since we have assumed that $p_{n}$ and $q_{n}$ are real, the zeros $z_{k}$ of $P(z)+Q(z)$ are real or occur in complex conjugate pairs. Accordingly, (9a) can be re-expressed as

$$
\begin{aligned}
& A_{1}(z)=\prod_{k=r+1}^{N}\left(\frac{z^{-1}-z_{k}^{-1}}{1-z^{-1} z_{k}^{-1}}\right) \\
& A_{2}(z)=\prod_{k=1}^{r}\left(\frac{z^{-1}-z_{k}}{1-z^{-1} z_{k}}\right) .
\end{aligned}
$$

Notice that all the poles of $A_{1}(z)$ and $A_{2}(z)$ are strictly inside the unit circle, and in turn are the zeros of $D(z)$. 
In view of (7) and (8), it follows that $G(z)+H(z)=$ $A_{1}(z)$ and $G(z)-H(z)=A_{2}(z)$. Thus, $G(z)$ and $H(z)$ are "complementary" with respect to $A_{1}(z)$ which is allpass. In addition, since the power-complementary property is satisfied, $G(z)$ and $H(z)$ form a "doubly-complementary" pair.

The transfer functions $G(z)$ and $H(z)$ can be implemented simply by implementing $A_{1}(z)$ and $A_{2}(z)$. Notice that any allpass function $A(z)$ of order $M$ can be implemented with only $M$ (rather than $2 M+1$ as in conventional dircct-form) multipliers [19]-[22]. Moreover, such an implementation of $A(z)$ can be made such that, in spite of multiplier quantization, $A(z)$ remains stable allpass [15] (i.e., a structurally lossless implementation [13]). Accordingly, $\left|G\left(e^{j \omega}\right)\right|$ and $\left|H\left(e^{j \omega}\right)\right|$ remain bounded above by unity for all $\omega$, even when the multipliers are quantized [15]. In other words, Fig. 1 represents a structurally passive implementation [13], and has low passband sensitivity. Moreover, the total number of multipliers required is $N-r+r=N$. But the implementation gives rise to two filters, $G(z)$ and $H(z)$; hence, we require $[N / 2]$ multipliers per transfer function. Recall that conventional direct-form implementation of $G(z)$ would require about $3[N / 2]$ multipliers. The number of delays in the allpassbased implementation of Fig. 1 can be kept at the minimum level $(=N)$, while at the same time using a total of only $N$ multipliers, simply by implementing $A_{1}(z)$ and $A_{2}(z)$ in the form of the one-multiplier Gray and Markel cascaded lattice structures [19]. Accordingly, the structure is dramatically efficient, and in addition has low passband sensitivity.

Now, if $P(z)$ and $Q(z)$ are both symmetric, the above allpass decomposition ((7)-(9)) does not hold, and hence the above efficient structure does not exist as such. However, there is a simple means of overcoming this problem when complex-arithmetic is permitted in the filter implementation. ${ }^{1}$

In order to see this, first recall that, by analytic continuation, (4) implies ${ }^{2}$

$$
\tilde{G}(z) G(z)+\tilde{H}(z) H(z)=1
$$

except at the poles. In terms of the polynomials $P(z)$, $Q(z)$, and $D(z)$, this implies

$$
\tilde{P}(z) P(z)+\tilde{Q}(z) Q(z)=\tilde{D}(z) D(z) .
$$

Next, with $P(z)$ and $Q(z)$ symmetric, we can write

$$
\tilde{P}(z)=z^{N} P(z) \quad \tilde{Q}(z)=z^{N} Q(z)
$$

whence (11) becomes

$$
P^{2}(z)+Q^{2}(z)=z^{-N} D\left(z^{-1}\right) D(z)
$$

\footnotetext{
${ }^{1}$ Notice that Fig. 1 involves only real multipliers, since $A_{1}(z)$ and $A_{2}(z)$ in (9) have real cocfficients.

${ }^{2}$ The tilde notation in general means that $z$ should be replaced with $z^{-1}$, and all complex coefficients conjugated. On the unit circle, the tilde notation is equivalent to complex conjugation of the entire function.
}

which can be decomposed as

$$
[P(z)+j Q(z)][P(z)-j Q(z)]=z^{-N} D\left(z^{-1}\right) D(z) .
$$

Let $\left\{z_{1}, z_{2}, \cdots, z_{N}\right\}$ denote the zeros of $D(z)$. None of these can be real for the following reason: If $z_{r}$ were a real zero of $D(z)$, then it would certainly be a zero of $P(z)+$ $j Q(z)$ or $P(z)-j Q(z)$. Assume, for example, that $P\left(z_{r}\right)+$ $j Q\left(z_{r}\right)=0$. Since $z_{r}$ is real and $P(z)$ has real coefficients, $P\left(z_{r}\right)$ is real, and so is $Q\left(z_{r}\right)$. Accordingly, we must have $P\left(z_{r}\right)=Q\left(z_{r}\right)=0$. In particular, this implies a common factor $\left(1-z^{-1} z_{r}\right)$ between $P(z)$ and $D(z)$, which has been ruled out earlier.

All $z_{k}$ 's above being complex occur in conjugate pairs $(D(z)$ being a polynomial with real coefficients). Hence

$$
D(z)=\prod_{k=1}^{M}\left(1-z^{-1} z_{k}\right)\left(1-z^{-1} z_{k}^{*}\right), \quad M=\frac{N}{2} .
$$

As a result, (14) can be rewritten as

$$
\begin{aligned}
& {[P(z)+j Q(z)][P(z)-j Q(z)]} \\
& \quad=z^{-N} \prod_{k=1}^{M}\left(1-z^{-1} z_{k}\right)\left(1-z^{-1} z_{k}^{*}\right)\left(1-z z_{k}\right)\left(1-z z_{k}^{*}\right) .
\end{aligned}
$$

Since $P(z)$ and $Q(z)$ are symmetric polynomials with real coefficients, the zeros of $P(z)+j Q(z)$ occur in reciprocal pairs, as do the zeros of $P(z)-j Q(z)$. Moreover, if $z_{k}$ is a zero of $P(z)+j Q(z)$, then its conjugate $z_{k}^{*}$ is a zero of $P(z)-j Q(z)$. In conclusion, the zeros shown on the right-hand side of (16) can be assigned as follows:

$$
\begin{aligned}
& P(z)+j Q(z)=\beta z^{-M} \cdot \prod_{k=1}^{M}\left(1-z^{-1} z_{k}\right)\left(1-z z_{k}\right) \\
& P(z)-j Q(z)=\beta^{*} z^{-M} \cdot \prod_{k=1}^{M}\left(1-z^{-1} z_{k}^{*}\right)\left(1-z z_{k}^{*}\right)
\end{aligned}
$$

where $\beta$ is a complex constant (to be identified soon). Dividing both sides of (17) and (18) by $D(z)$ in (15), we get

$$
\begin{aligned}
& G(z)+j H(z)=\beta \prod_{k=1}^{M} \frac{z^{-1}-z_{k}}{1-z^{-1} z_{k}^{*}} \\
& G(z)-j H(z)=\beta^{*} \prod_{k=1}^{M} \frac{z^{-1}-z_{k}^{*}}{1-z^{-1} z_{k}} .
\end{aligned}
$$

Thus, $G(z)$ and $H(z)$ can be written as linear combinations of stable allpass functions

$$
\begin{aligned}
& G(z)=\frac{1}{2}\left[\mathscr{A}_{1}(z)+\mathscr{A}_{2}(z)\right] \\
& H(z)=\frac{1}{2 j}\left[\mathscr{A}_{1}(z)-\mathscr{A}_{2}(z)\right]
\end{aligned}
$$

where

$$
\mathscr{A}_{1}(z)=\beta \prod_{k=1}^{M} \frac{z^{-1}-z_{k}}{1-z^{-1} z_{k}^{*}} \quad \mathscr{A}_{2}(z)=\beta^{*} \prod_{k=1}^{M} \frac{z^{-1}-z_{k}^{*}}{1-z^{-1} z_{k}} .
$$




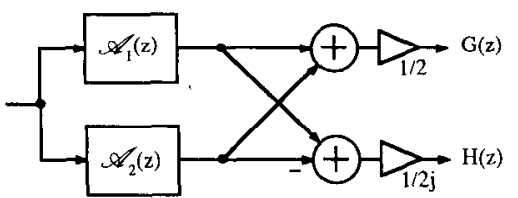

Fig. 4. Implementing the complementary pair.

Conversely

$$
\begin{aligned}
& \mathscr{A}_{1}(z)=G(z)+j H(z) \\
& \mathscr{A}_{2}(z)=G(z)-j H(z) .
\end{aligned}
$$

Note that $\mathscr{A}_{1}(z)$ and $\mathscr{A}_{2}(z)$ have complex coefficients, and that the coefficients of $\mathscr{A}_{2}(z)$ are the conjugates of the coefficients of $\mathscr{A}_{1}(z) . G(z)$ and $H(z)$ given by $(21)$ and (22), however, have real coefficients. In view of (24), we say that $G(z)$ and $j H(z)$ form an "allpass-complementary" pair. Since they also form a power-complementary pair (10), $G(z)$ and $j H(z)$ are said to form a doubly-complementary pair.

Fig. 4 represents the allpass decomposition scheme. Since the coefficients of $\mathscr{A}_{2}(z)$ are complex conjugates of those in $\mathscr{A}_{1}(z)$, it is only required to implement $\mathscr{A}_{1}(z)$. The real part of the filtered output sequence is the output corresponding to $G(z)$, whereas the imaginary part of the output sequence of $\mathscr{A}_{1}(z)$ corresponds to $H(z)$ as depicted in Fig. 2. Note that given any BR function $G(z)=$ $P(z) / D(z)$ with symmetric numerator $P(z)$, we can always obtain the implementation of Fig. 2 if there exists a BR function $H(z)=Q(z) / D(z)$ with symmetric numerator $Q(z)$, and such that (4), or equivalently, (11) holds. If the above conditions are all satisfied except that $Q(z)$ is antisymmetric, then the implementation of Fig. 4 can be obtained where the allpass functions now have real coefficients (and the factor $j$ is not required to obtain $H(z)$ ). The above-mentioned conditions on $G(z)$ and $H(z)$ are mild in the sense that a wide class of selective filters fall under this category. For example, Butterworth, Chebyshev, and elliptic digital IIR transfer functions satisfy the above requirements for all orders.

\section{A. On Computational Complexity of the Implementation}

With $N$ denoting the orders of $G(z)$ and $H(z)$, the allpass function $\mathscr{A}_{1}(z)$ in (23) has $N / 2$ first-order complex sections of the form

$$
\mathscr{A}_{1, k}(z)=\frac{z^{-1}-z_{k}}{1-z^{-1} z_{k}^{*}}
$$

where the poles $z_{k}$ are complex and of the form

$$
z_{k}=z_{k, r}+j z_{k, i}
$$

with $z_{k, r}$ and $z_{k, i}$ real. A schematic implementation of (26) is shown in Fig. 5(a). Since $w(n)$ is in general complex (even if $x(n)$ might be real), the circuit of Fig. 5(a) involves four real multiplications, which is equivalent to one complex multiplication. The function $\mathscr{A}_{1}(z)$ can thus be realized as a cascade of $M$ such sections, requiring a total of $M$ complex multipliers. In addition, if $\beta$ in (23)

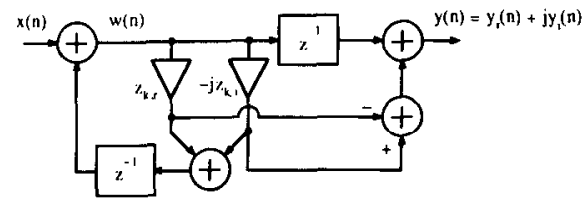

(a)

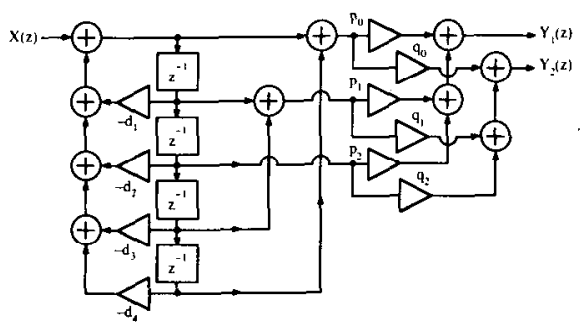

(b)

Fig. 5. (a) A first-order complex allpass section. (b) A direct-form implementation with $N$ delays and $2 N+2$ multiplicrs. $(N-4$ in the figure); $G(z)=Y_{1}(z) / X(z)$ and $H(z)=Y_{2}(z) / X(z)$.

operates on real input, this corresponds to two real multiplications. Thus, in Fig. 2, the number of real multipliers per transfer function is $N+1$. Each delay element in Fig. 5(a) stores both the real and imaginary parts; hence, a total of $4 M(=2 N)$ real storage elements is required (i.e., $N$ per transfer function).

A direct-form implementation of $G(z)$ and $H(z)$ (which share a common denominator) can be obtained with a total of $N$ delays and $2 N+2$ multipliers (i.e., $N / 2$ delays and $N+1$ multipliers per transfer function) as shown in Fig. 5(b). Thus, the number of multipliers is the same as for the new implementation, but there are fewer delays in the direct form. But the new implementation has exceptionally low passband sensitivity due to structural passivity, as we shall demonstrate.

\section{B. Comments on Pipelineability}

It is well known that a cascaded lattice implementation of a digital allpass function is very suitable for a high degree of pipelineability [31]. This comes about because of the unit delay element that isolates adjacent sections of a cascaded lattice structure. For details in this regard, we refer the reader to [31].

\section{The Design Procedure and a Design EXAMPI.F}

Given a BR function $G(z)=P(z) / D(z)$ as in (1) with symmetric $P(z)$, assume that there exists a $B R$ function $H(z)=Q(z) / D(z)$ satisfying (4) and such that $Q(z)$ is a symmetric polynomial. Then the necessary conditions for the allpass decomposition of (21) and (22) are satisfied and the pair $\{G(z), H(z)\}$ can be realized as in Fig. 2.

Since only $P(z)$ and $D(z)$ are known to begin with, $Q(z)$ remains to be found. This can be accomplished by computing an appropriate spectral factor of $\tilde{D}(z) D(z)-$ $\tilde{P}(z) P(z)$ by explicit root-finding, but such an elaborate procedure is not necessary. Since $Q(z)$ and $P(z)$ are symmetric, (12) and (13) hold and hence

$$
Q^{2}(z)=z^{-N} D\left(z^{-1}\right) D(z)-P^{2}(z) .
$$


Denote the right-hand side of (28), which is known, by $R(z)=\sum_{k-0}^{2 N} r_{k} z^{-k}$. Then the coefficients of $Q(z)$ can be easily computed recursively based on (28) as follows:

$$
\begin{aligned}
& q_{0}=\sqrt{r_{0}}, \quad q_{1}=r_{1} / 2 q_{0} \\
& q_{n}=\frac{r_{n}-\sum_{k=1}^{n-1} q_{k} q_{n-k}}{2 q_{0}}, \quad 2 \leqslant n \leqslant \frac{N}{2} \\
& q_{n}=q_{N-n}, \quad \frac{N}{2}<n \leqslant N .
\end{aligned}
$$

Once $Q(z)$ is found, we have to identify those zeros $z_{k}$ of $D(z)$ which should be associated with (17). This can be done by explicitly evaluating the roots of $P(z)+j Q(z)$ and thus identifying those that are inside the unit circle. The allpass function $\mathscr{A}_{1}(z)$ can now be implemented, provided the complex constant $\beta$ is identified. For this, notice that for $z=1$, (19) yields

$$
\beta \prod_{k=1}^{M} \frac{1-z_{k}}{1-z_{k}^{*}}=G(1)+j H(1)
$$

Since all quantities in (32) are known except $\beta$, we can identify the latter uniquely.

Example 1: As an example, consider the design of an 8th-order low-pass elliptic BR digital transfer function ${ }^{3}$
TABLE I

The Filter Coefficients in EXAMple 1

\begin{tabular}{rrrr}
\hline & \multicolumn{1}{c}{$\mathrm{p}_{\mathrm{n}}$} & \multicolumn{1}{c}{$\mathrm{q}_{\mathrm{n}}$} & \multicolumn{1}{c}{$\mathrm{d}_{\mathrm{n}}$} \\
\hline & & & \\
0 & 0.025913507 & 0.321023225 & 1.000000000 \\
1 & -0.028763702 & -1.901491193 & -3.982269600 \\
2 & 0.074164524 & 5.455303983 & $\mathbf{8 . 4 9 8 0 0 3 7 5 9}$ \\
3 & -0.041691969 & -9.724285248 & -11.486223632 \\
4 & 0.084194289 & 11.705785106 & 10.707331654 \\
5 & -0.041691969 & -9.724285248 & -6.906407027 \\
6 & 0.074164524 & 5.455303983 & 3.018707576 \\
7 & -0.028763702 & -1.901491193 & -0.809265853 \\
8 & 0.025913507 & 0.321023225 & 0.103727421 \\
\hline
\end{tabular}

response $\left|G\left(e^{j \omega}\right)\right|$ for the 4-bit allpass-based implementation of Fig. 4. The low passband sensitivity is evident from the plot of passband details, which are included in Fig. 7. Next, the same transfer function $G(z)$ was implemented in direct form with 9 bits per multiplier (i.e., nine nonzero binary digits in sign-digit code). The corresponding responses are shown in Fig. 8, which reveals a very poor passband sensitivity. Thus, a 4-bit allpass based implementation (Fig. 2) has a much better passband response than a 9-bit direct-form implementation. It should be noticed that the direct-form (rather than the standard cascade-form [21]) structure is being used for comparison purposes because the allpass function $\mathscr{A}_{1}(z)$ in the current example is being implemented in direct form.

$$
G(z)=\frac{P(z)}{D(z)}=\frac{0.026-0.029 z^{-1}+0.074 z^{-2}-0.042 z^{-3}+0.084 z^{-4}-0.042 z^{-5}+0.074 z^{-6}-0.029 z^{-7}+0.026 z^{-8}}{1-3.982 z^{-1}+8.498 z^{-2}-11.486 z^{-3}+10.707 z^{-4}-6.906 z^{-5}+3.019 z^{-6}-0.809 z^{-7}+0.104 z^{-8}} .
$$

Once $Q(z)$ is computed as outlined above, we have the complementary high-pass function

$$
H(z)=\frac{Q(z)}{D(z)}=\frac{0.321-1.901 z^{-1}+5.455 z^{-2}-9.724 z^{-3}+11.706 z^{-4}-9.724 z^{-5}+5.455 z^{-6}-1.901 z^{-7}+0.321 z^{-8}}{1-3.982 z^{-1}+8.498 z^{-2}-11.486 z^{-3}+10.707 z^{-4}-6.906 z^{-5}+3.019 z^{-6}-0.809 z^{-7}+0.104 z^{-8}} .
$$

We then compute the zeros of $P(z)+j Q(z)$, and thus identify $\mathscr{A}_{1}(z)$ as

$$
\mathscr{A}_{1}(z)=\beta \prod_{k=1}^{4} \frac{z^{-1}-z_{k}}{1-z^{-1} z_{k}^{*}}
$$

where

$$
\begin{gathered}
\beta=0.4698+j 0.8828 \\
z_{1}=0.4344-j 0.2253 \quad z_{2}=0.4831+j 0.5675 \\
z_{3}=0.5244-j 0.7367 \quad z_{4}=0.5492+j 0.8075 .
\end{gathered}
$$

In order to demonstrate the low passband sensitivity properties, the structure of Fig. 2 was simulated on a digital computer with quantized multipliers. The allpass function $\mathscr{A}_{1}(z)$ was implemented in direct form rather than by cascading the first-order sections of the form in Fig. 5; each multiplier $z_{k r}$ and $z_{k i}$ was quantized to four nonzero binary digits in sign-digit code.

Fig. 6 shows the magnitude responses of $H(z)$ and $G(z)$ in the ideal (i.e., unquantized) case. Fig. 7 shows the

\footnotetext{
${ }^{3}$ Note that the coefficients displayed in (33) and (34) are heavily quantized for convenience. Table I displays a more accurate version of these coefficients.
}

\section{Comment on Stopband Sensitivity}

As such, with structurally passive implementations, good passband sensitivity does not necessarily imply good stopband sensitivity. This has been observed earlier [15]. In particular, a parallel combination of two allpass structures (which is the overall form of the new structures we have reported) does not necessarily have low stopband sensitivity. This situation is also true with wave lattice digital filters [4]. However, one can perform a discrete-space optimization on the finite-wordlength coefficients in order to maximize the stopband attenuation. Since the passband sensitivity is low, this optimization hardly affects the passband.

\section{LATTICE STRUCTURES FOR IMPLEMENTATION OF COMPleX Allpass Functions}

For digital allpass functions with real coefficients, a number of cascaded lattice structures are available for implementation [12], [19], [20]. Such structures are known to have low noise, and can be designed to be free of limit cycle oscillations under zero input [12]. In addition, some 


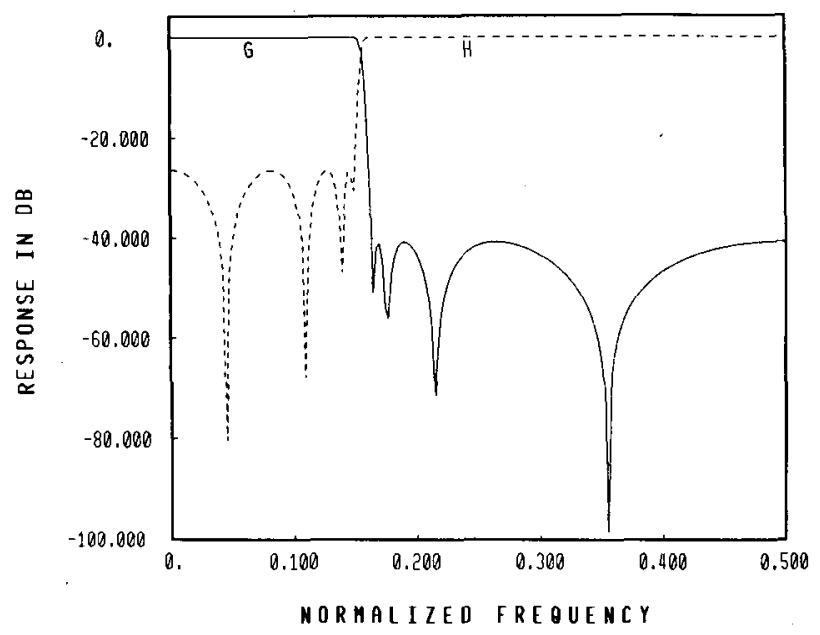

Fig. 6. Example 1. The 8th-order complementary pair response.

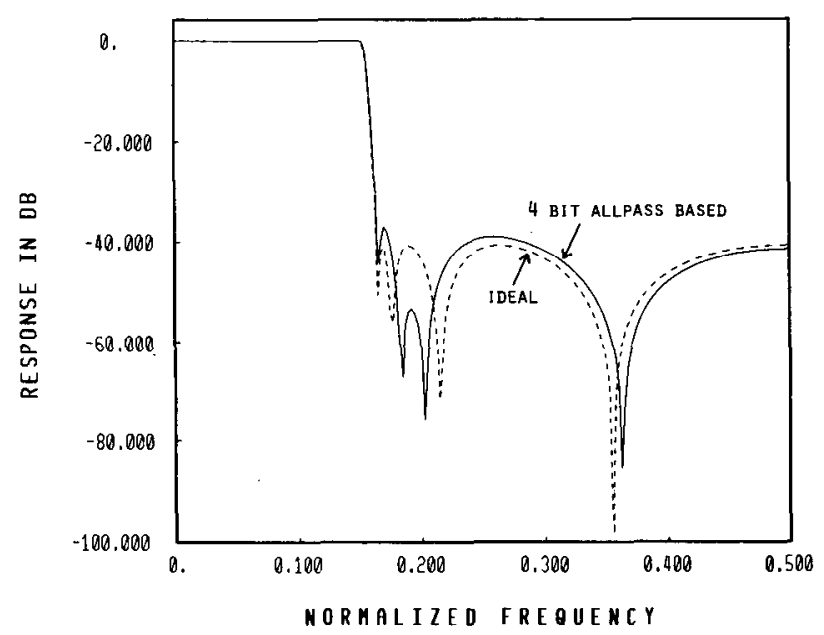

(a)

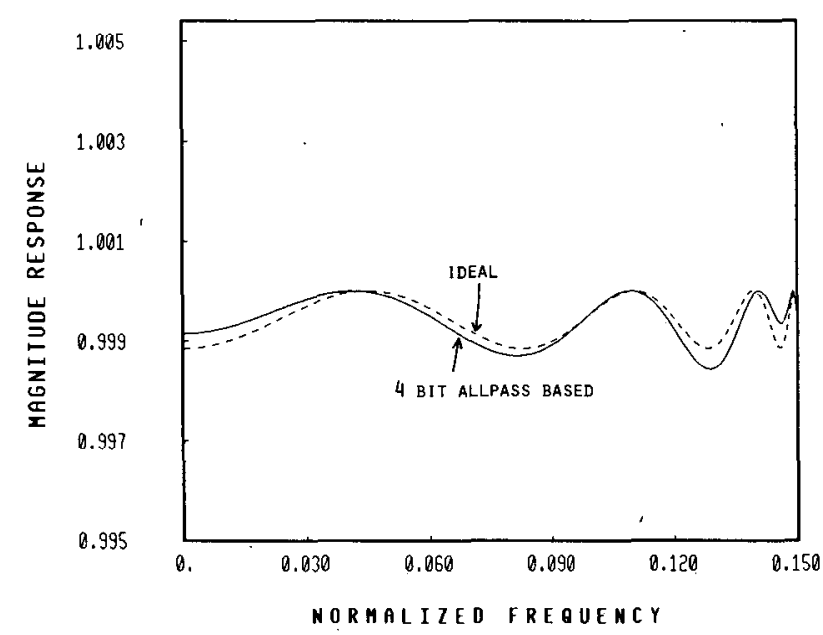

(b)

Fig. 7. (a) Example 1. The 4-bit complex-allpass based implementation. (In this paper, $b$-bit implementation means that each real multiplier is replaced by a sum of $b$ powers of 2 using canonic sign-digit codes). (b) Example 1. Passband details for 4-bit complex-allpass based implementation.

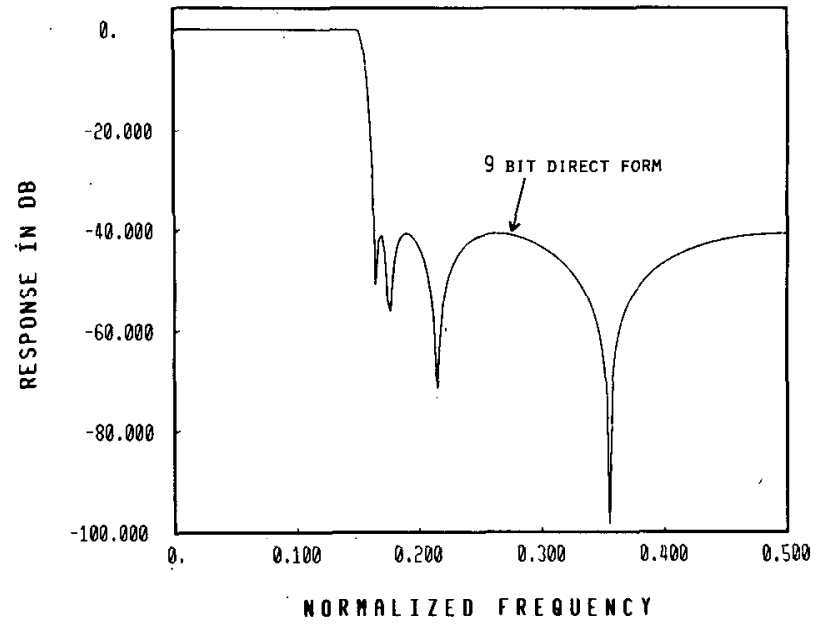

(a)

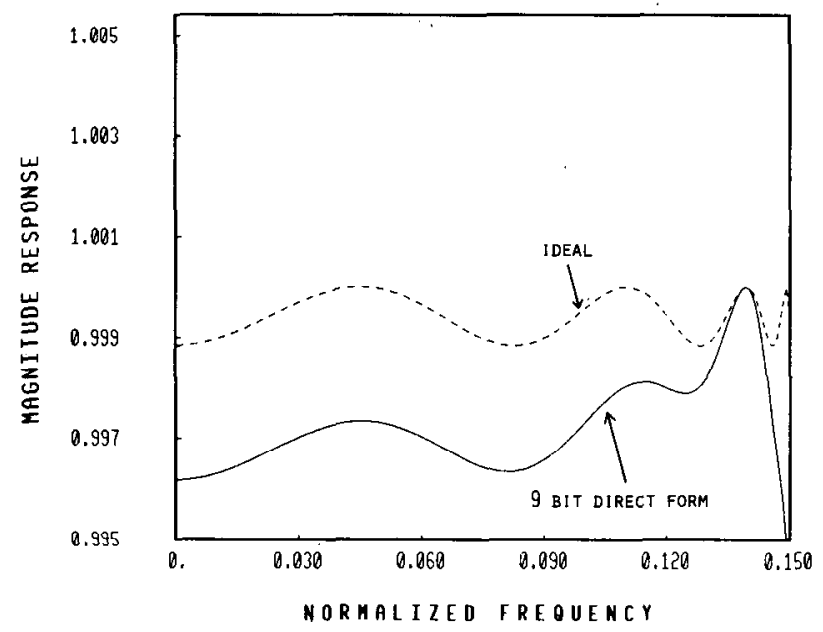

(b)

Fig. 8. (a) Example 1. The 9-bit direct-form implementation. (b) Example 1. Passband details for direct-form implementation with 9 bits.

of these structures have automatic internal scaling properties (normalized structures [20]).

Most of these lattice structures can be generalized for the case where the allpass functions have complex coefficients. In order to see how a lattice structure can be generated for the complex allpass function

$$
G_{m}(z)=\frac{d_{m, m}^{*}+d_{m, m-1}^{*} z^{-1}+\cdots+z^{-m}}{1+d_{m, 1} z^{-1}+\cdots+d_{m, m} z^{-m}}
$$

consider the recursive relation

$$
z^{-1} G_{m-1}(z)=\frac{G_{m}(z)-k_{m}^{*}}{1-k_{m} G_{m}(z)}
$$

with $k_{m}$ chosen as

$$
k_{m}=G_{m}^{*}(\infty)=d_{m, m} .
$$

Assuming that $G_{m}(z)$ is a stable allpass function (of order $m$ ), it can be verified that $\left|k_{m}\right|<1$ and that $G_{m-1}(z)$ is a stable allpass function of order $m-1$. Once the order reduction implied by (39) is accomplished, we can construct $G_{m}(z)$ from $G_{m-1}(z)$ as shown in Fig. 9. 


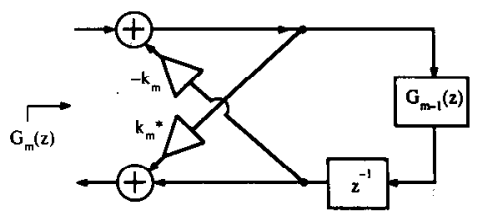

Fig. 9. Implementation of $G_{m}(z)$ in terms of $G_{m-1}(z)$.

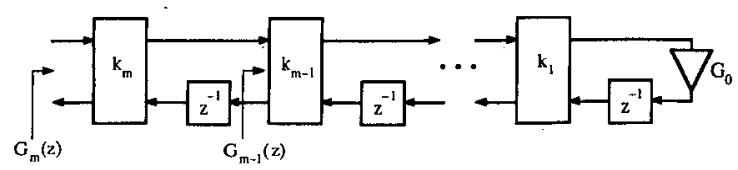

Fig. 10. The cascaded complex lattice for $G_{m}(z)$.

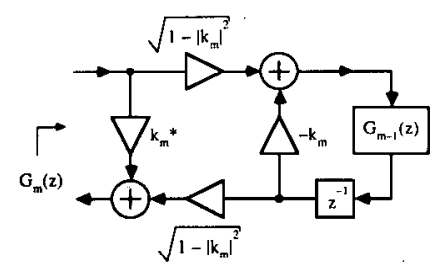

Fig. 11. The normalized complex lattice.

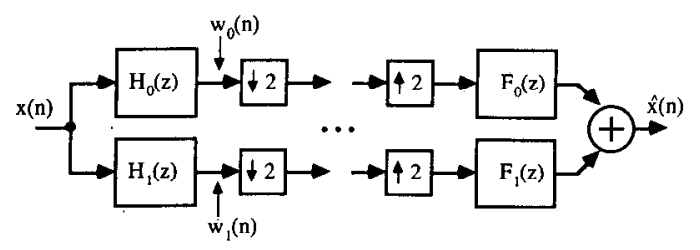

Fig. 12. The quadrature mirror filter bank.

If the above order-reduction process is now repeated, we obtain a cascaded lattice realization of $G_{m}(z)$ as shown in Fig. 10 where each building block is a lattice section as in Fig. 9. The multiplier $G_{0}$ is in general complex, but has unit magnitude.

A normalized lattice structure can be obtained by noticing that the relation between $G_{m}(z)$ and $G_{m-1}(z)$ in Fig. 9 remains unaltered by rearranging the lattice as shown in Fig. 11.

\section{Multirate (Quadrature Mirror) FILTERING APPLICATIONS}

A well-known bank of filters used in signal-coding and communications applications is the quadrature-mirror filter bank [23]-[25] shown in Fig. 12. Here $x(n)$ is an input signal with spectral occupancy $0 \leqslant \omega \leqslant \pi . I_{0}(z)$ and $H_{1}(z)$ are low-pass and high-pass filters, respectively, operating on $x(n)$. The filtered signals $w_{0}(n)$ and $w_{1}(n)$ are decimated in time, and then transmitted after possible coding. The received signals are decoded, interpolated, and passed through filters $F_{0}(z)$ and $F_{1}(z)$, and then recombined to form $\hat{x}(n)$. The set of filters $\left\{H_{0}(z), H_{1}(z)\right\}$ is called the analysis bank, whereas the pair $\left\{F_{0}(z), F_{1}(z)\right\}$ forms the synthesis bank.

One of the main requirements in the scheme of Fig. 12 is to be able to make $\hat{x}(n)$ "as close" to $x(n)$ as possible. In general, $\hat{x}(n)$ suffers from three types of errors: aliasing (due to undersampling after nonideal filtering), amplitude

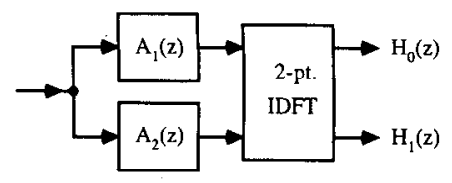

Fig. 13. Implementing $H_{0}(z)$ and $H_{1}(z)$.

distortion, and phase distortion. It is well known [23]-[25], [30] that aliasing effects can be completely eliminated if the transfer functions $H_{0}(z), H_{1}(z), F_{0}(z)$, and $F_{1}(z)$ are related as follows:

$$
F_{0}(z)=H_{0}(z) \quad F_{1}(z)=-H_{1}(z) \quad H_{1}(z)=H_{0}(-z) .
$$

With aliasing-effects removed, the system of Fig. 12 is a shift-invariant (and of course, linear) system with transfer function [24]

$$
T(z) \triangleq \frac{\hat{X}(z)}{X(z)}=\frac{1}{2}\left[H_{0}^{2}(z)-H_{1}^{2}(z)\right] .
$$

If $H_{0}(z)$ and $H_{1}(z)$ are linear-phase FIR filters, there is no phase distortion; thus, the goal is to make $T(z)$ resemble a delay as closely as possible so as to minimize the amplitude distortion.

If, on the other hand, $H_{0}(z)$ and $H_{1}(z)$ are IIR functions, the amplitude distortion can be completely eliminated, provided $T(z)$ is forced to be allpass. (Phase distortion, however, remains in this case, but can be equalized by allpass cascade equalizers.) In this section, we consider only IIR QMF banks. Accordingly, our goal is to force the distortion transfer function $T(z)$ in (41) to be allpass, so that there is no amplitude distortion.

Since the allpass-based structures of Figs. 1 and 2 require a much smaller number of multipliers (particularly for odd $N$ ) compared to other implementations, and since they exhibit low passband sensitivity, we wish to design the analysis bank $\left\{H_{0}(z), H_{1}(z)\right\}$ in this form. Thus, referring to Fig. 13, we wish to have ${ }^{4}$

$$
H_{0}(z)=\frac{A_{1}(z)+A_{2}(z)}{2} \quad H_{1}(z)=\frac{A_{1}(z)-A_{2}(z)}{2} .
$$

Recall from Section II that if $N$ is odd, and if the numerators of $H_{0}(z)$ and $H_{1}(z)$ are symmetric and antisymmetric, respectively, then the implementation of Fig. 13 is possible, provided

$$
\left|H_{0}\left(e^{j \omega}\right)\right|^{2}+\left|H_{1}\left(e^{j \omega}\right)\right|^{2}=1 .
$$

For such an implementation, we thus have

$$
\begin{aligned}
& A_{1}(z)=H_{0}(z)+H_{1}(z) \\
& A_{2}(z)=H_{0}(z)-H_{1}(z)
\end{aligned}
$$

where $A_{1}(z)$ and $A_{2}(z)$ are allpass functions with real coefficients. It is clear from (44) and (45) that

$$
H_{0}^{2}(z)-H_{1}^{2}(z)=A_{1}(z) A_{2}(z)
$$

\footnotetext{
${ }^{4}$ In Figs. 13-16, we have used the IDFT building blocks to represent the operations in (42) because this reveals the relation to the general form of polyphase uniform DFT filter banks [23].
} 


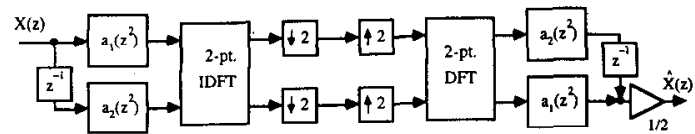

Fig. 14. The complete QMF bank implementation.

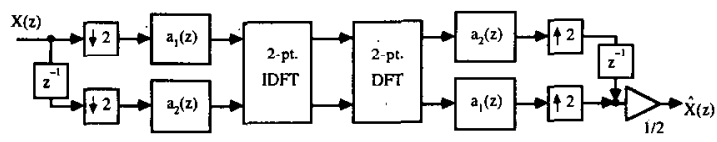

Fig. 15. The simplified structure.

which is allpass. In other words, the distortion function $T(z)$ in (41) is automatically allpass, and there is no amplitude distortion. Thus, the allpass-based implementation of Fig. 13 is a natural and ideal candidate for the QMF application.

In summary, we have the following important result: let $H_{0}(z)=P(z) / D(z)$ and $H_{1}(z)=Q(z) / D(z)$ be odd-order BR transfer functions such that $P(z)$ is symmetric and $Q(z)$ antisymmetric, and such that (43) holds. Then the filter bank $\left\{H_{0}(z), H_{1}(z)\right\}$ can be implemented as in Fig. 13. Moreover, if it is employed in the QMF structure of Fig. 12 with (40) holding, then not only is aliasing perfectly cancelled, but also the amplitude distortion is completely eliminated.

Recall from Section II that $A_{2}(z)$ has order $r$ and $A_{1}(z)$ has order $N-r$. Next, in view of the relation $H_{1}(z)=$ $H_{0}(-z)$ in (40), we have from (44) and (45)

$$
\begin{aligned}
& A_{1}(z)=2 h_{0}+2 h_{2} z^{-2}+\cdots=a_{1}\left(z^{2}\right) \\
& A_{2}(z)=2 z^{-1}\left[h_{1}+h_{3} z^{-2}+\cdots\right]=z^{-1} a_{2}\left(z^{2}\right)
\end{aligned}
$$

where $\left\{h_{k}\right\}$ represents the impulse response corresponding to $H_{0}(z)$. As a result of this form, $A_{1}(z)$ and $A_{2}(z)$ can be written as

$$
\begin{aligned}
& A_{1}(z)=\prod_{k=1}^{(N-r) / 2} \frac{\alpha_{k}^{*}+z^{-2}}{1+\alpha_{k} z^{-2}} \\
& A_{2}(z)=z^{-1} \prod_{k=1}^{(r-1) / 2} \frac{\beta_{k}^{*}+z^{-2}}{1+\beta_{k} z^{-2}} .
\end{aligned}
$$

Such allpass sections have also been used in the context of transmultiplexer design by Constantinides and Valenzuela [27], [28]. Thus, the implementation of Fig. 13 actually requires only $(N-1) / 2$ multiplications, i.e., $[(N-1) / 4]$ per transfer function of order $N$.

Since $F_{0}(z)$ and $F_{1}(z)$ in Fig. 12 are related to the analysis-bank filters $H_{0}(z)$ and $H_{1}(z)$ as in (40), the synthesis bank can be obtained by transposing the analysis bank. Fig. 14 shows the complete implementation. In view of the fact that $a_{1}\left(z^{2}\right)$ and $a_{2}\left(z^{2}\right)$ are functions of $z^{-2}$, we can move the decimators and interpolators [23] and obtain the implementation of Fig. 15, which immediately leads to the polyphase structure [23], [29] of Fig. 16.

Before proceeding to the complex case (i.e., $N=$ even), we summarize the salient features of the implementation of Fig. 16. This figure represents a QMF analysis-synthesis

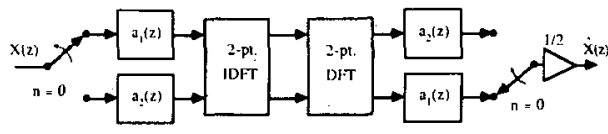

Fig. 16. The polyphase implementation.

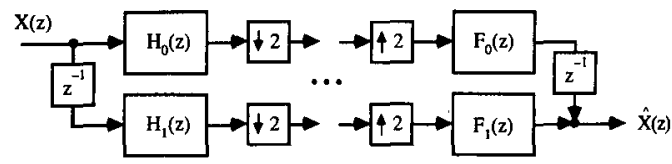

Fig. 17. The modified QMF bank.

system, and is free from aliasing and amplitude distortions. The overall distortion-transfer function $T(z)$ is alIpass, and given by

$$
T(z)=\frac{1}{2} A_{1}(z) A_{2}(z)=\frac{1}{2} z^{-1} a_{1}\left(z^{2}\right) a_{2}\left(z^{2}\right) .
$$

There are four transfer functions of order $N$ involved in the entire filter bank, and we require $[(N-1) / 4]$ multiplications per transfer function. The coefficients of $a_{1}\left(z^{2}\right)$ and $a_{2}\left(z^{2}\right)$ are all real. Finally, the transfer functions $H_{0}(z), H_{1}(z)$ exhibit very low passband sensitivity with respect to the multiplier coefficients.

Next consider the case where $H_{0}(z)$ and $H_{1}(z)$ in Fig. 12 have even-order $N$. For such cases, we cannot obtain the allpass-based implementation represented by Fig. 13. Instead, we can use complex allpass sections, as discussed in Section II. If $H_{0}(z)$ and $H_{1}(z)$ satisfy all the requirements of Section II so that they can be implemented as in Fig. 2, then the condition analogous to (24) and (25) holds in particular. In other words

$$
\mathscr{A}_{1}(z)=H_{0}(z)+j H_{1}(z) \quad \mathscr{A}_{2}(z)=H_{0}(z)-j H_{1}(z)
$$

whence

$$
H_{0}^{2}(z)+H_{1}^{2}(z)=\mathscr{A}_{1}(z) \mathscr{A}_{2}(z) .
$$

Thus, in place of (46), which is satisfied for odd $N$, we have (53) holding true for even $N$. However, the QMF bank of Fig. 12 requires (46) rather than (53) to hold, so the implementation of the bank $\left\{H_{0}(z), H_{1}(z)\right\}$ in the form of Fig. 2 is not compatible with the requirements of Fig. 12.

This difficulty can be overcome by employing the modified QMF bank proposed in [25] by Galand and Nussbaumer. Fig. 17 shows the modified scheme, which incorporates two additional delays. In the circuit of Fig. 17, aliasing effects are cancelled by the choice

$$
F_{0}(z)=H_{0}(z) \quad F_{1}(z)=H_{1}(z) \quad H_{1}(z)=H_{0}(-z) .
$$

Once aliasing is thus cancelled, we have [25]

$$
T(z) \triangleq \frac{\hat{X}(z)}{X(z)}=\frac{1}{2} z^{-1}\left[H_{0}^{2}(z)+H_{1}^{2}(z)\right] .
$$

In other words, in order to eliminate amplitude distortion, it is required to force $H_{0}(z)$ and $H_{1}(z)$ to be such that 
$T(z)$ in (55) is allpass. But this is precisely what the complex-allpass-based implementation of Fig. 2 accomplishes, as shown by (53). In summary, for even $N$, the allpass-based implementation of Fig. 2 automatically satisfies the condition required by the QMF bank of Fig. 17 , for eliminating amplitude distortion.

Since $H_{1}(z)$ is equal to $H_{0}(-z)$, we have, by (52)

$\mathscr{A}(z)=H_{0}(z)+j H_{0}(-\dot{z}) \quad \mathscr{A}_{*}(z)=H_{0}(z)-j H_{0}(-z)$

where $\mathscr{A}(z)$ denotes $\mathscr{A}_{1}(z)$, and $\mathscr{A}_{*}(z)$ is obtained from $\mathscr{A}(z)$ by conjugating the coefficients of $\mathscr{A}(z)$. From (56), it can be verified that

$$
\mathscr{A}(-z)=j \mathscr{A}_{*}(z)
$$

which shows that if $\alpha$ is a pole of $\mathscr{A}(z)$, then $-\alpha^{*}$ is also a pole of $\mathscr{A}(z)$. Consequently, $\mathscr{A}(z)$ can be expressed as ${ }^{5}$

$$
\begin{array}{r}
\mathscr{A}(z)=\eta \prod_{k=1}^{n_{1}}\left(\frac{-\alpha_{k}^{*}+z^{-1}}{1-\alpha_{k} z^{-1}}\right)\left(\frac{\alpha_{k}+z^{-i}}{1+\alpha_{k}^{*} z^{-1}}\right) \\
\cdot \prod_{l=1}^{n_{2}}\left(\frac{-j \beta_{l}+z^{-1}}{1+j \beta_{l} z^{-1}}\right)
\end{array}
$$

where $N / 2=2 n_{1}+n_{2}$. In (58), $\beta_{l}$ are real numbers, whereas $\alpha_{k}$ are complex. The quantity $\eta$ is a complex constant with $|\eta|=1$. Each first-order allpass-section involving $\beta_{l}$ can be implemented with one real multiplier. Each second-order section involving $\alpha_{k}$ can be implemented with only two real multipliers, as can be seen by writing the section transfer function as

$$
\frac{-\left|\alpha_{k}\right|^{2}+z^{-1}\left(\alpha_{k}-\alpha_{k}^{*}\right)+z^{-2}}{1-\left(\alpha_{k}-\alpha_{k}^{*}\right) z^{-1}-\left|\alpha_{k}\right|^{2} z^{-2}}=\frac{C_{0}+j z^{-1} C_{1}+z^{-2}}{1-j z^{-1} C_{1}+C_{0} z^{-2}}
$$

where $C_{0}$ and $C_{1}$ are real constants.

By using the constraint (57) in the general expression (58), it can be verified that $\eta$ satisfies the relation

$$
\arg \eta= \pm \pi / 4 \text {. }
$$

Accordingly

$$
\eta=\frac{1}{\sqrt{2}}(1 \pm j)
$$

If the scale factor $1 / \sqrt{2}$ in (61) is ignored, $\eta$ can be implemented in a multiplierless form. Accordingly, the only significant multiplications in the implementation of $\mathscr{A}(z)$ are due to $\alpha_{k}$ 's and $\beta_{l}$ 's in (58). In summary, the analysis bank, which can be realized by implementing $\mathscr{A}(z)$, requires a total of $N / 2$ real multipliers; these multipliers, in general, operate on complex internal signals.

The synthesis filters $F_{0}(z), F_{1}(z)$ are related to the analysis filters as in (54). It can therefore be verified that $F_{0}(z)$ and $F_{1}(z)$ can be implemented as shown in Fig. 18. The real signals $y_{0}(n-1)$ and $y_{1}(n)$ (which are inputs to $F_{0}(z)$

\footnotetext{
${ }^{5}$ See Appendix for more discussions in this connection.
}

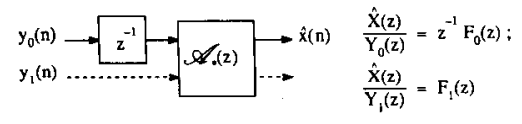

Fig. 18. The synthesis-bank implementation; the signal $y_{0}(n-1)$ is the real part of the input to $\mathscr{A}_{*}(z)$, and $y_{1}(n)$ is the corresponding imaginary part. The real part of the output of $\mathscr{A}_{*}(z)$ is the reconstructed signal $\hat{x}(n)$.

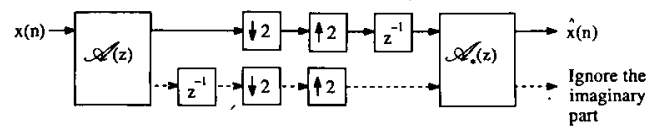

(a)

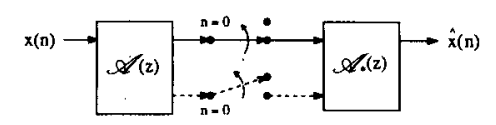

(b)

Fig. 19. (a) The complete QMF bank, with complex allpass filters in the analysis and synthesis sections. Solid lines represent real parts of signals and broken lines represent imaginary parts. (b) A commutator-switch model for Fig. 19(a). The switches are out of phase, so that the effects of the delay unit $\left(z^{-1}\right)$ in Fig. 19(a) are accounted for.

and $F_{1}(z)$, respectively) are combined into a complex signal $y_{0}(n-1)+j y_{1}(n)$ and this complex signal is taken as the input to $\mathscr{A}_{*}(z)$. The real part of the output is then equal to $\hat{x}(n)$. The complete QMF bank, implemented in terms of $\mathscr{A}(z)$ and $\mathscr{A}_{*}(z)$ is shown in Fig. 19(a), and can be redrawn as in Fig. 19(b). Notice that Fig. 19(b) implicitly incorporates the delay $z^{-1}$ (which precedes $H_{1}(z)$ and $\left.F_{0}(z)\right)$, and the decimation/interpolation operations. The entire QMF bank can be implemented using $N$ real multipliers (i.e., $[N / 4]$ per transfer function), where each multiplier, in general, operates on a complex signal.

\section{A. Relation to Earlier Work}

The recursive digital filters $H_{0}(z)$ and $H_{1}(z)$ in (42) take on the form $H_{0}(z)=P(z) / D_{1}\left(z^{2}\right), H_{1}(z)=$ $Q(z) / D_{1}\left(z^{2}\right)$, in view of the special forms of $A_{1}(z)$ and $A_{2}(z)$ in (49) and (50). The problem of approximating a desired magnitude response, with a rational function whose denominator is a function of $z^{-2}$, has been handled in the past [32].

The idea of splitting a signal into two bands based on two power-complementary transfer functions (i.e., satisfying (43)) has been considered earlier in [33]. Next, Barnwell [34] has indicated how IIR QMF banks can be constructed so as to cancel aliasing and at the same time eliminate amplitude distortion (i.e., force $T(z)$ to be allpass). However, to the best of our knowledge, the double complementarity of two IIR transfer functions has not hitherto been exploited to obtain the alias-free structure of Figs. 13-16. Notice that double complementarity implies (43) and in addition (44) and (45), and this is what ensures that $T(z)$ is allpass. The use of wave digital filters in the QMF bank is discussed in [26], where it is shown that aliasing and amplitude distortion can be eliminated. Such results can also be explained based on the results presented above. Finally, we believe that the splitting of a band into two signals based on a complex allpass function, and subse- 
quent recombination without aliasing and with no amplitude distortion, has not been observed earlier.

\section{CONCLUding REMARKS}

A new class of IIR digital filter structures has been presented, which can be used for efficient and low-sensitive implementation of several useful transfer functions. The conditions required to be satisfied by the transfer functions, so as to be implemented in the proposed form, are mild. Thus, Butterworth, Chebyshev, and elliptic digital filters belong to the class of transfer functions that can be implemented in this manner. In addition to their low sensitivity, the structures are ideally suited for quadrature mirror filter applications.

For the case of even $N$, the allpass function involved in the implementation is complex. Fig. 5 represents an implementation of a first-order section with one complex multiplier but with two (complex) delay units. It remains to be explored whether first-order complex allpass sections can be implemented with one complex multiply and one complex delay. Finally, in connection with the lattice sections of Figs. 9-11, it is worth exploring whether each section can be rearranged to have one multiplier (complex) and one delay. (For allpass functions with real coefficients, such lattice structures with one multiplier and one delay per section are well known [12], [19].)

\section{APPENDIX}

For most IIR filters, the transfer function $G(z)$ has the property that $G(z) G\left(z^{-1}\right)$ can be expressed as

$$
G(z) G\left(z^{-1}\right)=\frac{1}{1+\epsilon^{2} R(z) R\left(z^{-1}\right)}
$$

where $R(z)$ is, in general, a rational function. An appropriate choice of $R(z)$ gives rise to Butterworth, Chebyshev, and elliptic filters as special cases [21]. For elliptic low-pass filters, $R(z)$ has the form

$$
R(z)=\left(\frac{1-z^{-1}}{1+z^{-1}}\right)^{\prime} \prod_{k=1}^{m} \frac{\left(1-z^{-1} e^{j \theta_{k}}\right)\left(1-z^{-1} e^{-j \theta_{k}}\right)}{\left(1-z^{-1} e^{j \omega_{k}}\right)\left(1-z^{-1} e^{-j \omega_{k}}\right)}
$$

where the quantity $l$ is zero for even-order filters and unity for odd-order filters. ${ }^{6}$ Here, $\theta_{k}$ and $\omega_{k}$ are the reflection and transmission zeros, respectively [35], of $G(z)$. Two elliptic low-pass filters having the same order and same band edges $\omega_{p}$ and $\omega_{S}$ have the same set of reflection and transmission zeros; they differ only in terms of $\epsilon$, which controls the ripples.

It can be verified that $R(z)$ satisfies

$$
R\left(z^{-1}\right)=(-1)^{l} R(z)
$$

Now, if the passband and stopband edges $\omega_{p}$ and $\omega_{S}$ are

\footnotetext{
${ }^{6}$ Notice that, according to our definitions of $\epsilon$ and $R(z)$, the quantity $1 / \sqrt{1+c^{2}}$ does not necessarily correspond to maximum passband attenuation.
}

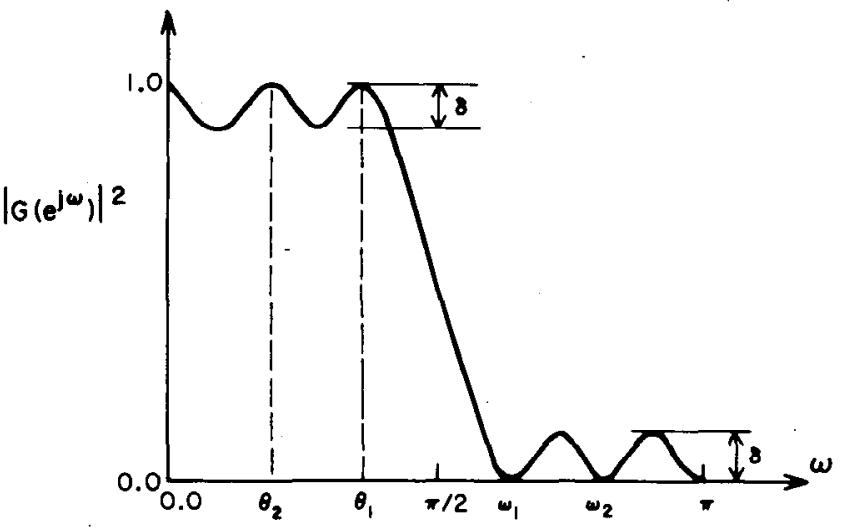

Fig. 20. Plot of the magnitude-squared function of a low-pass elliptic filter exhibiting symmetry with respect to $\pi / 2$.

symmetric with respect to $\pi / 2$, we have

$$
\omega_{p}+\omega_{s}=\pi \text {. }
$$

If we define $K(z)$ to be such that

$$
K(z) K\left(z^{-1}\right)=1-G(-z) G\left(-z^{-1}\right)
$$

then $K(z)$ has reflection zeros $\pi-\omega_{k}$ and transmission zeros $\pi-\theta_{k}$. Moreover, in view of (A4), $K(z)$ is a low-pass elliptic filter with same band edges as $G(z)$; hence, we have the relation

$$
\theta_{k}+\omega_{k}=\pi
$$

As a result, it can be verified that $R(z)$ satisfies

$$
R(z)=1 / R(-\bar{z})
$$

Now, if the plot of $\left|G\left(e^{j \omega}\right)\right|^{2}$ exhibits a symmetry about $\pi / 2$ (as in Fig. 20), then we can see that $K(z)$ is precisely the same as $G(z)$. By using (A1) and (A5), we can then show that $\epsilon$ is unity. Accordingly, at a pole of $G(z)$, we have the relation

$$
1+R(z) R\left(z^{-1}\right)=0 .
$$

In view of the relations (A3) and (A7), we thus have, at a pole of $G(z)$

$$
1+(-1)^{l} \frac{R(z)}{R(-z)}=0 .
$$

Since $G(z)$ is lowpass with passband edge $\omega_{p}<\pi / 2$, we have $\theta_{k}<\pi / 2$; hence, the zeros of $R(z)$ are strictly in the right half of the $z$-plane, and the poles are strictly in the left half plane. So $T(z)=R(z) / R(-z)$ has all poles strictly in the left half plane, and satisfies $|T(j y)|=1$ for all real $y$. Thus, by employing the maximum modulus theorem, we arrive at

$$
|T(z)| \begin{cases}=1, & \text { for } z=j y \\ <1, & \text { for } \operatorname{Re}(z)>0 \\ >1, & \text { for } \operatorname{Re}(z)<0 .\end{cases}
$$

It immediately follows that all solutions of (A9) lie on the imaginary axis of the $z$-plane. In summary, if $G(z)$ is a low-pass elliptic filter whose magnitude-squared response has symmetry with respect to $\pi / 2$ as in Fig. 20 , then all the poles of $G(z)$ are on the imaginary axis. 
The main point here is that, in the QMF application, since $H_{0}(z)$ and $H_{1}(z)$ are related by (43), where $H_{1}(z)=$ $H_{0}(-z)$, the response $\left|H_{0}\left(e^{j \omega}\right)\right|^{2}$ does satisfy the symmetry displayed in Fig. 20. Accordingly, (58) gets simplified because $n_{1}=0$. The factors involving $\beta_{l}$ can be implemented using one complex delay and one real multiplier only. The real multiplier, in general, operates upon complex signals.

\section{ACKNOWLEDGMENT}

The authors wish to thank Dr. Y. Neuvo of the Tampere University of Technology, Finland, for useful discussions.

\section{REFERENCES}

[1] A. Fettweis, "Digital' filter structures related to classical filter networks," Arch. Elek. Übertrangungs., vol. 25, pp. 79-81, Feb. 1971.

[2] A. Fettweis, "Pseudopassivity, sensitivity, and stability of wave digital filter," IEEE Trans. Circuit Theory, vol. CT-19, pp. 668-673, Nov. 1972.

[3] M. N. S. Swamy and K. Thyagarajan, "A new type of wave digital filters," $J$. Franklin Inst., vol. 300, no. 1, pp. 41-58, July 1975 .

[4] A. Fettweis, "Wave digital lattice filters," Int. J. Circuit Theory Appl., vol. 2, pp. 203-211, June 1974.

[5] S. S. Lawson and A. G. Constantinides, "The design of digital filters using linear transformations on classical LC two-ports," in Proc. Network Theory Symp., 1975, pp. 369-378.

[6] .P. DeWilde and E. Deprettere, "Orthogonal cascade realization of real multiport digital filters," Int. J. Circuit Theory Appl., vol. 8, pp. $245-277,1980$.

[7] D. Henrot and C. T. Mullis, "A modular and orthogonal digital filter structure for parallel processing," in Proc. IFEE Int. Conf. Acoustics, Speech, Signal Process., Apr. 1983, pp. 623-626.

[8] S. K. Rao and T. Kailath, "Orthogonal digital filters for VLSI implementation," IEEE Trans. Circuits Syst., vol. CAS-31, pp. 933-945, Nov. 1984.

[9] J. Szczupak and S. K. Mitra, "On digital filter structures with low coefficient sensitivities," Proc. IEEE, vol. 66, pp. 1082-1083, Sept. 1978.

[10] R. C. Agarwal and C. S. Burrus, "New recursive digital filter structures having very low sensitivity and roundoff noise," IEEE Trans. Circuits Syst., vol. CAS-22, pp. 921-926, Dec. 1975.

[11] A. Fettweis and K. Meerkotter, "Suppression of parasitic oscillations in wave filters," IEEE Trans. Circuits Syst., vol. CAS-22, pp. 239-246, Mar. 1975.

[12] A. H. Gray, Jr., "Passive cascaded lattice digital filters," IEEE Trans, Circuits Syst., vol. CAS-27, pp. 337-344, May 1980.

[13] P. P. Vaidyanathan and S. K. Mitra, "Low passband sensitivity digital filters: A generalized viewpoint and synthesis procedures," Proc. IEEE, pp. 404-423, Apr. 1984.

[14] P. P. Vaidyanathan and S. K. Mitra, "Very low-sensitivity FIR filter implementation using "structural passivity' concept," IEEE Trans. Circuits Syst., vol. CAS-32, pp. 360-364, Apr. 1985.

[15] P. P. Vaidyanathan, S. K. Mitra, and Y. Neuvo, "A new approach to the realization of low sensitivity IIR digital filters," IEEE Trans. Acoustics, Speech, Signal Process., vol. ASSP-34, pp. 350-361, Apr. 1986.

[16] P. P. Vaidyanathan and S. K. Mitra, "Passivity properties of low sensitivity digital filter structures," IEEE Trans. Circuits Syst., vol. CAS-32, pp. 217-224, Mar. 1985.

[17] P. P. Vaidyanathan, "The discrete-time bounded real lemma in digital filtering," IEEE Trans. Circuits Syst., vol. CAS-32, pp. 918-924, Sept. 1985.

[18] R. Ansari and B. Liu, "A class of low-noise computationally efficient recursive digital filters with applications to sampling rate alterations," IEEE Trans. Acoustics, Speech, Signal Process., vol. ASSP-33, pp. 90-97, Feb. 1985.

[19] A. H. Gray and J. D. Markel, "Digital lattice and ladder filter synthesis," IEEE Trans. Audio Electroacoustics, vol. AU-21, pp. 491-500, Dec. 1973.

[20] A. H. Gray, Jr. and J. D. Markel, "A normalized digital filter structure," IEEE Trans. Acoustics, Speech, Signal Process., vol. ASSP-23, no. 3, pp. 268-277, June 1975.

[21] A. V. Oppenheim and R. W. Schafer, Digital Signal Processing. Englewood Cliffs, NJ: Prentice-Hall, 1975.

[22] S. K. Mitra and K. Hirano, "Digital all-pass networks," IEEE Trans. Circuits Syst., vol. CAS-21, no. 5, pp. 688-700, Sept. 1974.

[23] R. E. Crochiere and L. R. Rabiner, Multirate Digital Signal Processing. Englewood Cliffs, NJ: Prentice-Hall, 1983.
[24] V. K. Jain and R. E. Crochiere, "Quadrature mirror filter design in the time domain," IEEE Trans. Acoustics, Speech, Signal Process., vol. ASSP-32, pp. 353-361, Apr. 1984.

[25] C. R. Galand and H. J. Nussbaumer, "New quadrature mirror filter structures," IEEE Trans. Acoustics, Speech, Signal Process., vol. ASSP-32, pp. 522-531, June 1984.

[26] A. Fettweis, J. A. Nossek, and K. Meerkotter, "Reconstruction of signals after filtering and sampling rate reduction," in Proc. IEEE Int. Conf. Acoustics, Speech, Signal Process., (San Diego, CA), Mar. 1974, pp. 11.7.1-11.7.4.

[27] A. G. Constantinides and R. A. Valenzuela, "A class of efficient interpolators and decimators with applications in transmultiplexers," in Proc. IEEE Int. Symp. Circuits Syst., (Rome, Italy), May 1982, pp. 260-263.

[28] A. G. Constantinides and R. A. Valenzuela, "An efficient and modular transmultiplexer design," IEEE Trans. Commun., vol. 30, pp. 1629-1641, July 1982.

[29] M. G. Bellanger, G. Bonnerot, and M. Coudreuse, "Digital filtering by polyphase network: Application to sample-rate alteration and filter banks," IEEE Trans. Acoustics, Speech, Signal Process., vol. ASSP-24, pp. 109-114, Apr. 1976.

[30] A. Croisier, D. Esteban, and C. Galand, "Perfect channel-splitting by use of interpolation/decimation/tree decomposition techniques," presented at Int. Conf. Information Sci. Syst., Patras, Greece, 1976.

[31] P. DeWilde, E. Deprettere, and R. Nouta, "Parallel and pipelined VLSI implementation of signal processing algorithms," in VLSI and Modern Signal Processing, S. Y. Kung, H. J. Whitehouse, and T. Kailath, Prentice-Hall, Eds. Englewood Cliffs, NJ: 1985.

[32] H. G. Martinez and T. W. Parks, "A class of infinite-duration impulse response digital filters for sampling-rate reduction," $I E E E$ Trans. Acoustics, Speech; Signal Process., vol. ASSP-27, pp. 154-162, Apr. 1979.

[33] T. A. Ramstad, "Branch filtering using FIR and IIR complementary structures," private communication.

[34] T. P. Barnwell III, "Subband coder design incorporating recursive quadrature filters and optimum ADPCM coders," IEEE Trans. Acoustics, Speech, Signal Process., vol. ASSP-30, pp. 751-765, Oct. 1982.

[35] A. S. Sedra and P. O. Brackett, Filter Theory and Design: Active and Passive. Champaign, IL: Matrix Publishers, 1978.

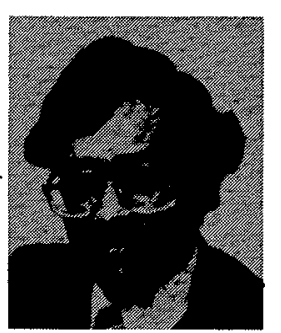

P. P. Vaidyanathan (S'80-M'83) was born in Calcutta, India, on October 16, 1954. He received the B.Sc. (with honors) degree in physics, 2nd the B. Tech and $M$. Tech degrees in radiophysics and electronics from the University of Calcutta, India, in 1974, 1977, and 1979, respectively, and the Ph.D. degree in electrical and computer engineering from the University of California, Santa Barbara, in 1982.

He was a Postdoctoral Fellow at the University of California, Santa Barbara, from September 1982 to February 1983. Since March 1983, he has been with the California Institute of Technology, Pasadena, as an Assistant Professor of Electrical Engineering. His main research interests are in digital signal processing, linear systems, and filter design.

Dr. Vaidyanathan serves as the Vice-Chairman of the Technical Program committee for the 1983 IEEE International Symposium on Circuits and Systems. He currently serves as an Associate Editor for the IEEE TRANSACTIONS ON Circuits AND SySTEMS. He was the recipient of the Award for Excellence in Teaching at the California Institute of Technology for $1983 / 84$. He was also a recipient of NSF's Presidential Young Investigator Award, in 1986. 


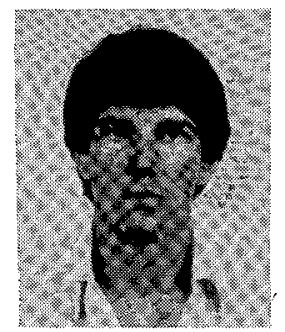

Phillip A. Regalia (S'86) was born in Walnut Creek, CA, in 1962. He received the B.Sc. (highest honors) in electrical engineering from the University of California at Santa Barbara in 1985.

Presently, he is a Ph.D. candidate at UCSB, where be works as a research assistant in the Signal Processing Laboratory. His research interests include analog circuit theory and digital signal processing.

幽

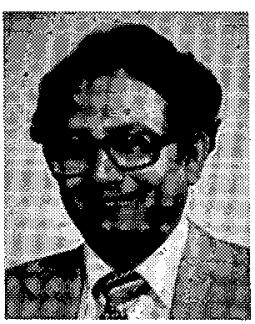

Sanjit K. Mitra (S'59-M'63-SM'63-SM'69F'74) received the B.S. (Hons.) degree in physics from the Utkal University, India, in 1953, the M.Sc. (Tech.) degree in radio physics and electronics from the University of Calcutta, India, in 1956, and the M.S. and Ph.D. degrees in electrical engineering from the University of California, Berkeley, in 1960 and 1962, respectively.

He was a member of the faculty of the Cornell University, Ithaca, NY, from 1962 to 1965 , and a member of technical Staff of the Bell Laboratories from 1965 to 1967 . He joined the faculty of the University of California, Davis, in 1967 and transferred to the Santa Barbara (UCSB) campus in 1977 as a Professor of Electrical and Computer Engineering. From July 1979 to June 1982, he served as the Chairman of the Department of Electrical and Computer Engineering at UCSB. He is a consultant to the Lawrence Livermore National Laboratory, Livermore, CA and the Los Alamos National Laboratory, Los Alamos, NM and is the
Consulting Editor for the Electrical/Computer Science and Engineering Series of the Van Nostrand Reinhold Company, NY. He has also served as a consultant to Ampex Corporation, Fairchild Semiconductor, Siliconix, HDR Sciences, the U.S. Army, General Motors Delco Division, and the World Bank. He has held visiting appointments at the Indian Institute of Technology, New Delhi, India; Kobe University, Japan; University of Erlangen-Nuernberg, West Germany, the Australian National University, Canberra, Australia; Tampere University of Technology, Tampere, Finland; the Federal University of Rio de Janeiro, Brazil, and the University of Zagreb, Yugoslavia. Dr. Mitra has published a number of papers in active and passive networks and digital filters, and is the author of Analysis and Synthesis of Linear Active Networks and An Introduction to Digital and Analog Integrated Circuits and Applications and Editor of Active Inductorless Filters, Co-Editor of Modern Filter Theory and Design (with G. C. Temes), and Two-Dimensional Digital Signal Processing (with M. P. Ekstrom). He holds two patents in active filters.

Dr. Mitra is a Fellow of the American Association for the Advancement of Science and a member of the American Society for Engineering Education, Sigma Xi, and Eta Kappa Nu. He is a member of the Advisory Board of the George R. Brown School of Engineering of the Rice University, Houston, TX, and an Advisory Professor of the Northern Jiaotong University, Beijing, China. He was an Associate Editor of the IEEE Transactions on Circuits and Systems, and served on the editorial boards of the Proceedings of the IEEE and IEEE Press, and presently is on the editorial board of Circuits, Systems, and Signal Processing. He has been a member of the Administrative Committee of the IEEE Circuits and Systems Society, Local Arrangements Chairman (1969), General Chairman (1974), and Program Chairman (1983) of the Annual IEEE International Symposium on Circuits and Systems and is presently the Past-President of the IEEE Circuits and Systems Society. He is the recipient of the 1973 F. E. Terman Award and 1985 AT\&T Foundation Award of the American Society of Engineering Education, a Visiting Professorship from the Japan Society for Promotion of Science in 1972, and, Fulbright Distinguished Lecturer Award for Brazil in 1984, and for Yugoslavia in 1986. 